\title{
Yeast telomeres: how to ignore essential double-strand DNA breaks?
}

\author{
Lubomír Tomáška ${ }^{1}$, Judita Sadovská ${ }^{1}{\text { Jozef } \text { Nosek }^{2} \text { and Jack D. Griffith }}^{3}$ \\ ${ }^{1}$ Department of Genetics, Faculty of Natural Sciences, Comenius University, Mlynska dolina B-1, 84215 \\ Bratislava, Slovakia \\ ${ }^{2}$ Department of Biochemistry, Faculty of Natural Sciences, Comenius University, Mlynska dolina CH-1, 84215 \\ Bratislava, Slovakia \\ ${ }^{3}$ Lineberger Comprehensive Cancer Center and Department of Microbiology and Immunology, University of \\ North Carolina, Chapel Hill, NC 27599, USA
}

\begin{abstract}
Summary
DNA looping is one of the mechanisms involved in telomere maintenance. It probably provides a solution not only to 'the end-replication problem', but also for the protection of chromosomal ends against degradation enzymes and, as typical double-strand breaks, from DNA repair machinery. Telomeric loops (t-loops) formed by an invasion of protruding 3' overhangs into the doublestranded telomeric regions were observed in a variety of organisms ranging from ciliates to mammals. Genetic data indicate that looping also occurs at the telomeres of Saccharomyces cerevisiae, suggesting its importance for telomere function in yeast. However, several observations argue against the presence of 'true' t-loops in the budding yeast telomeres (e.g. the lack of TRFlike protein, heterogeneous telomeric sequences). Instead, telomeres in S. cerevisiae appear to form fold-back structures mediated by protein-protein interactions. To directly visualize the telomeric structure in budding yeast, we developed a system based on a mini-chromosome carrying an array of $l a c$ operator sequences allowing its purification by the lac repressor affinity column. In contrast to budding yeast, the fission yeast Schizosaccharomyces pombe contains a homologue of the human telomeric protein TRF2, designated Taz1p. As the TRF2 protein has been implicated in remodelling telomeres into t-loops, the ability of Tazlp to promote t-loop formation is examined by electron microscopy using purified protein and synthetic templates containing a double-stranded fission yeast telomeric tract. Our studies should shed some light not only on telomeric architecture in yeast, but should also be instrumental in deciphering detailed telomeric structure in higher eukaryotes.
\end{abstract}

Keywords: telomere - t-loop - Taz1 - fission yeast - budding yeast - chromatin - silencing

\section{INTRODUCTION}

Telomeres, the DNA-protein complexes at the ends of linear chromosomes, stabilize the termini and protect them from end-to-end fusion. With a few exceptions, telomeres of most eukaryotic cells consist of an array of short repeats rich in guanines on the strand running from 5 ' to 3 ' towards the chromosome end. Another general feature of nuclear telomeres is the presence of a 3' overhang of the G-rich strand at the termini (McEachern et al. 2000).
The exciting studies on the replication of eukaryotic chromosome ends resulted in the formulation of the 'telomere hypothesis', suggesting a key role for telomere dynamics in the process of tumorigenesis (Fig. 1). According to this hypothesis, telomere shortening accompanied by decapping is considered a tumor suppression mechanism and the re-activation of telomerase - a specialized nucleoprotein enzyme that extends the chromosome tips, thus compensating the losses of terminal sequences encountered during replication is an essential step in the origin and infinite growth 
of immortal cell lines (Bodnar et al. 1998, Greider 1999). Based on this scenario, the telomerase represents a promising target for anti-cancer therapy and the telomerase inhibitors might become a reasonable and effective therapeutic tool (Hahn et al. 1999, Raymond et al. 1996). However, the situation seems to be far more complicated and the telomere hypothesis has recently been revisited (Autexier and Greider 1996, Holt et al. 1996). It has been demonstrated that telomerase-negative human tumors and tumor-derived immortal cell lines, acquired very long telomeric blocks through unknown telomere-lengthening mechanisms. The maintenance of telomeres by alternative, telomerase-independent, mechanisms might be important in certain types of cancer, and in therapeutic attempts, the emergence of such mechanisms may represent a potential source of resistance of tumor cells toward the telomerase inhibitors (Biessmann and Mason 1997, Bryan et al. 1997, Bryan et al. 1995, Bryan et al. 1997, Bryan and Reddel 1997, Colgin and Reddel 1999, Raymond et al. 1996, Reddel et al. 2001, Reddel et al. 1997).

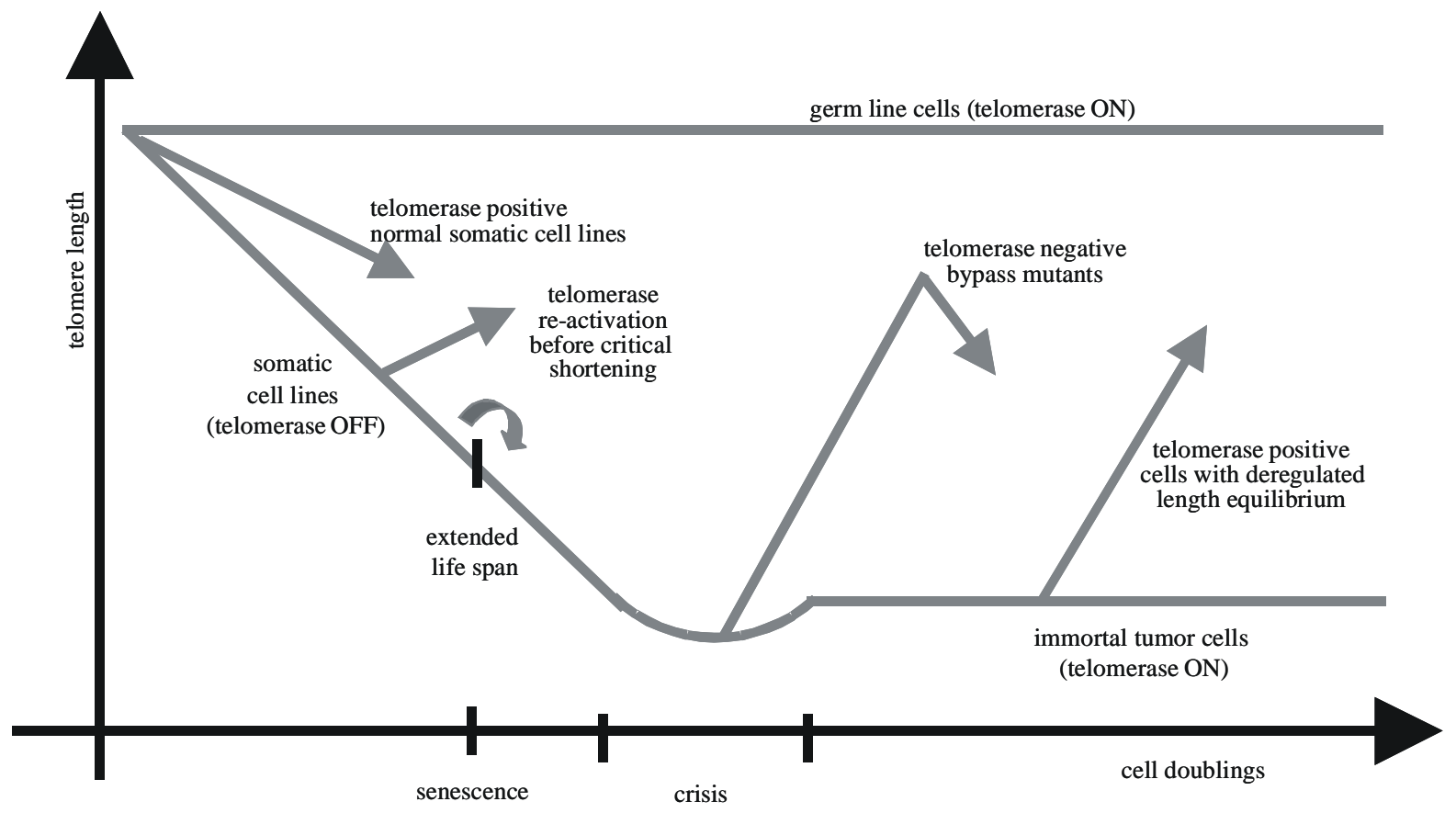

Fig. 1. The telomere/telomerase hypothesis (according to Autexier and Greider 1996).

Although telomerase-independent mechanisms of telomere elongation in human immortal cells are well documented, their nature is not yet understood. The analysis of telomerase-independent mechanisms in lower eukaryotes has resulted in several important observations. For example, $S$. cerevisiae mutants with defects in the telomerase component revealed that yeasts reconstitute chromosomal ends by a RAD52-dependent recombination mechanism (Wang and Zakian 1990). By analogy, a recombinational mechanism was proposed also for mouse cells with a knockedout gene for the telomerase component (Blasco et al. 1997, Lansdorp, 1997). Recently, Reddel and co-workers identified a RAD52-dependent recombination as the major pathway for restoring telomeres in telomerase-negative cells (Dunham et al. 2000).

However, recombination might not be the only way of survival. Schizosaccharomyces pombe mutants in the gene coding for telomerase RNA solve the problem either by a recombinational RAD52-dependent mechanism or by circularization of their chromosomes (Naito et al. 1998, Nakamura et al. 1998). Moreover, rare survivors of Kluyveromyces lactis with the double mutation $\Delta$ terl $\Delta$ rad52 might suggest the existence of yet another mechanism (McEachern and Blackburn 1996). In several organisms that include insect (Chironomus, Anopheles, Drosophila) and plant (Allium) species, a telomerase-independent strategy of telomere elongation naturally operates as a primary mechanism (Biessmann and Mason 1997). Although in many cases the unequal recombinational mechanism was proposed, Drosophila melanogaster maintains the ends of chromosomes by the repeated transfer of telomereassociated retrotransposons (Levis et al. 1993, Pardue et al. 1996, Pardue et al. 1997). 

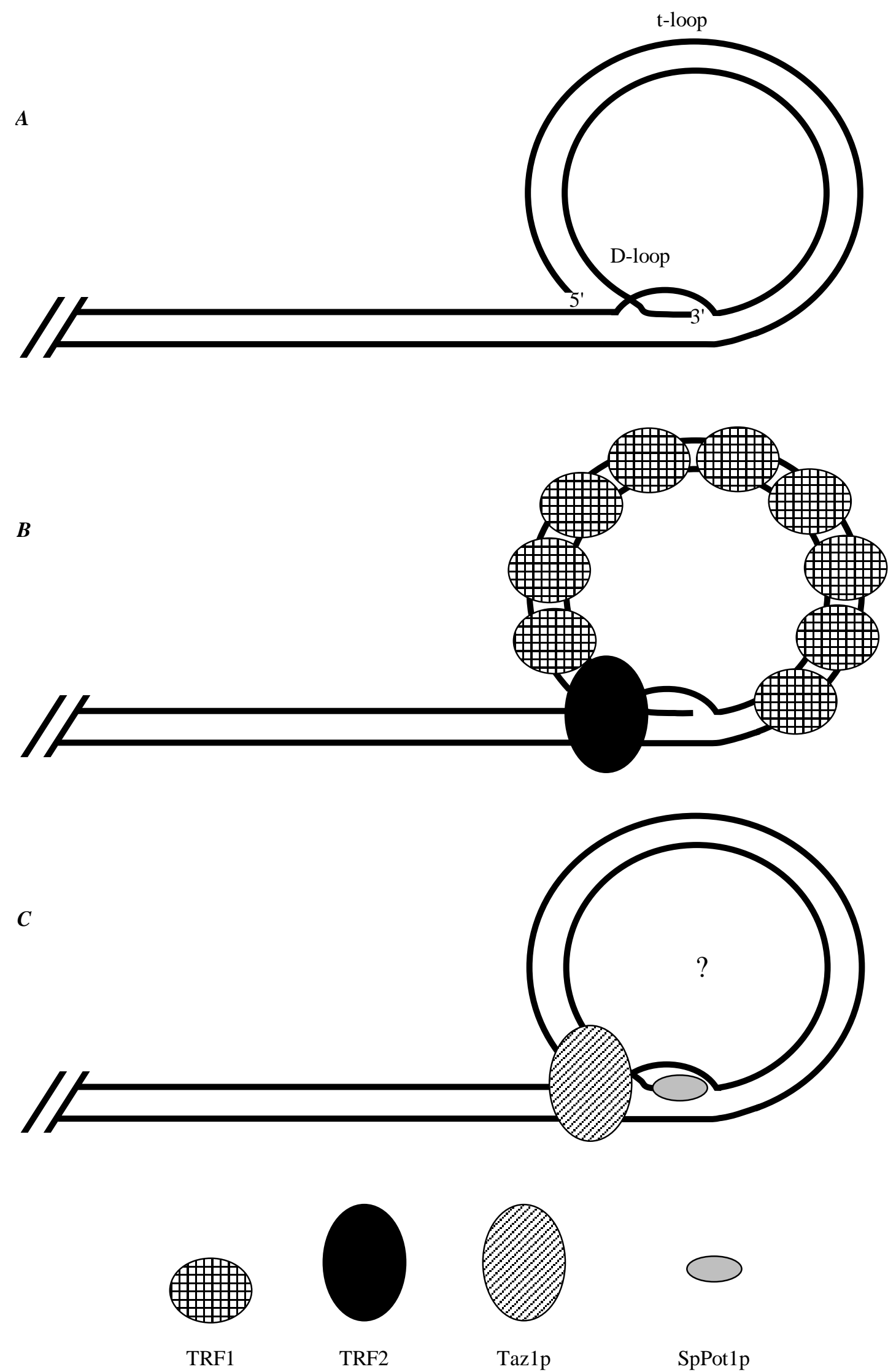

Fig. 2. A putative structure of capped telomere based on a t-loop. (Griffith et al., 1999) (A) Architecture of the t-loop. 3' single-stranded overhang invades a double-stranded telomeric region forming a D-loop. $(B)$ Position TRF1 and TRF2 within the mammalian t-loop. Additional telomeric proteins, like Pot1p, Rap1p, Est1p, Rad50p, Mre11p, Nbs1p, Ku, and tankyrase, are not shown. $(C)$ Hypothetical t-loop at fission yeast telomere. 


\section{TELOMERES - IGNORED DOUBLE-STRAND BREAKS}

Telomeres exist in at least two different states (Blackburn 2000). The 'open' complex is probably needed for the telomerase to access the end of the telomeric DNA. The 'closed' complex caps and protects chromosome ends not only against degradation by nucleases, but importantly, against protein complexes mediating repair of doublestrand breaks. In fact, loss of telomere capping seems to be one of the reasons for chromosomal end-to-end fusions and genomic instability (Desmaze et al. 2003). The changes in telomere architecture mediated by specific protein components of the telomeric chromatin are essential for proper functioning of the chromosomal ends (Rhodes et al. 2002).

The 'closed' state of the telomere is exemplified by the telomeric loops (t-loops) formed by an invasion of the 3' single-stranded (ss) overhang of mammalian telomeres into the duplex telomeric region (Griffith et al. 1999) (Fig. 2A). T-loops were subsequently found at the termini of micronuclear chromosomes of Oxytricha nova (Murti and Prescott 1999), at the telomeres of Trypanosoma brucei (Munoz-Jordan et al. 2001), as well as at the ends of linear mitochondrial DNA of the yeast Candida parapsilosis (Tomaska et al. 2002). It was suggested that t-loops would effectively hide the natural end of the chromosome to protect it from the DNA repair machinery. In addition, the 3' overhang mediated priming of the new DNA synthesis, followed by appropriate cleavages and gap filling, could give rise to a large increase in telomere size. In the original study on mammalian t-loops it was demonstrated that the formation of loops is mediated by a protein TRF2 (Griffith et al. 1999). TRF2 and its homologue TRF1 were identified as proteins binding to double-stranded (ds) telomeric DNA in the form of homodimers (Bilaud et al. 1997, Broccoli et al. 1997, Chong et al. 1995). Both TRF1 and TRF2 function to negatively regulate telomere length, as overexpression of either protein leads to the progressive shortening of the telomeric tract over many cellular divisions (Smogorzewska et al. 2000, van Steensel and de Lange 1997). Although TRF1 and TRF2 exhibit a high degree of homology, their role in telomere dynamics seems to be different, probably due to the differences at their N-termini (Karlseder 2003, Karlseder et al. 2002) (Fig. 2B). In contrast to TRF2, TRF1 is unable to promote t- loop formation in vitro. Whereas TRF1 forms filamentous structures on telomeric repeat arrays and promotes parallel pairing of telomeric tracts in vitro (Griffith et al. 1998), TRF2 induces t-loop formation and binds preferentially at the ss/ds junction at the 3' telomeric overhang (Stansel et al. 2001). A 5'-TTAGGG-3' overhang of at least six nucleotides is required for the loop formation. Termini with 5' overhangs or blunt ends were deficient in the loop formation. On the other hand, the addition of non-telomeric sequences to the distal portion of a 3' overhang beginning with TTAGGG repeats, only modestly diminishes looping (Stansel et al., 2001).

Several nuclear proteins including Tin2 (Kim et al. 1999), tankyrase (Smith et al. 1998), and hRap1 (Li et al. 2000) associate with telomeric DNA via binding to TRF1 and TRF2 and thus modulate their activities including the t-loop formation. Other proteins, not directly associated with TRF1 and TRF2, may affect the t-loop dynamics. For example, Pot1 protein binds with a high affinity to the telomeric 3' single-stranded overhang (Baumann and Cech 2001) and can play a role in both recruiting telomerase to the chromosomal ends (Evans and Lundblad 1999) and affecting the ability of 3' telomeric overhang to invade the double-stranded region of the telomere. In addition, protein components of the nonhomologous endjoining pathway $(\mathrm{Ku}, \mathrm{Mre11}, \mathrm{Nbs} 1, \mathrm{Xrs} 2$ and Rad50) are essential constituents of the telosome (Chan and Blackburn 2002). This leads to a paradox: telomeres, typical double-strand DNA breaks, are kept intact partly due to the presence of proteins essential for a repair of the same type of DNA structures within internal parts of the genome. At the telomere, these proteins seem to target the chromosome ends for the action of telomerase as opposed to non-homologous endjoining. One possible solution to this apparent contradiction is that even though telomeres are double-strand DNA breaks, their higher-order architecture makes them resistant to the DNA repair machinery. Telomeric loops are obvious candidates for such structural design. If telomere looping provides a solution to the above problem, then it should be a general feature of chromosomal ends, including those in lower eukaryotes, like yeasts. The aim of our study is to test this hypothesis in both the budding yeast Saccharomyces cerevisiae and the fission yeast Schizossacharomyces pombe. 

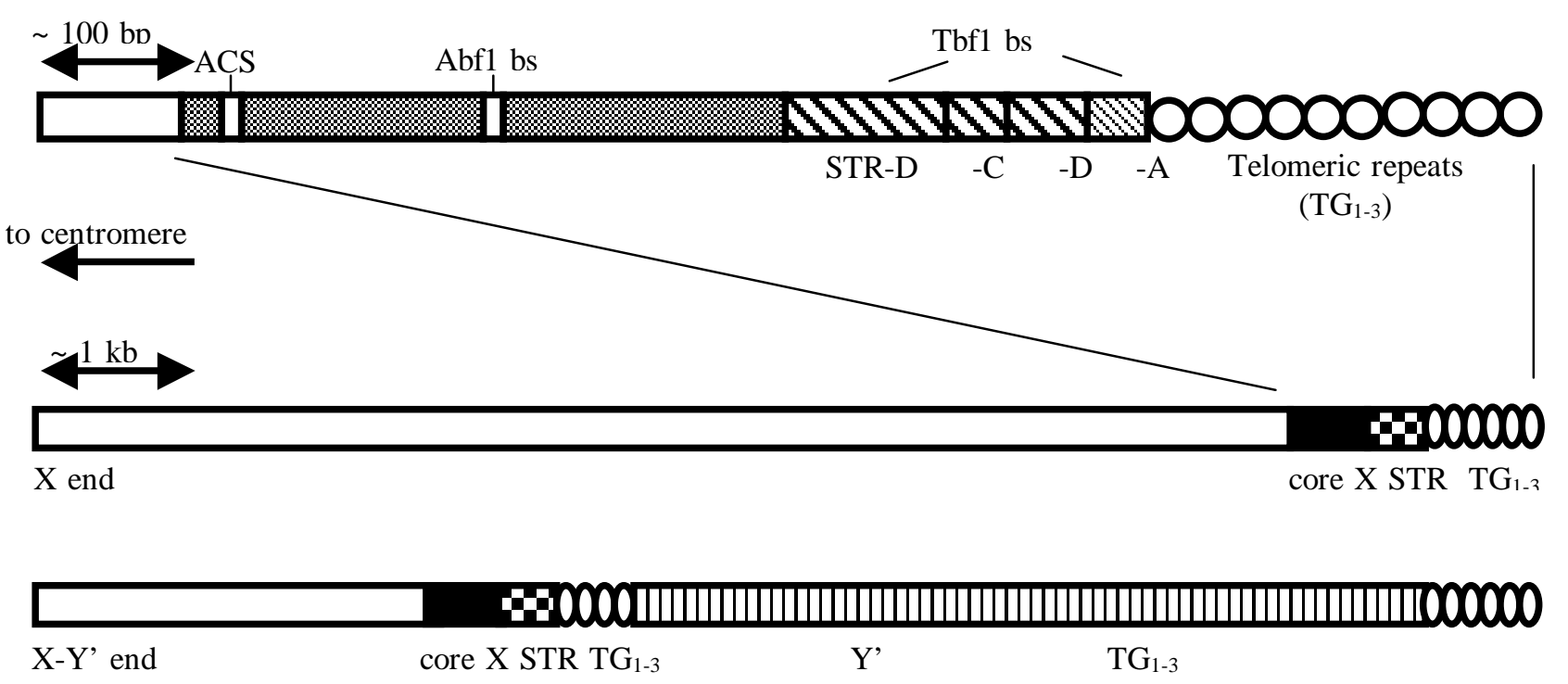

Fig. 3. General structure of budding yeast telomeres (adapted from http://www.le.ac.uk/ge/ej112/research/ telostruc/ X_Y_description.html). Shown are both X end (containing only X element) and X-Y' end (containing one X and one to four $\mathrm{Y}^{\prime}$ elements). All 32 chromosomal ends in S. cerevisiae contain an X element (composed of a core X element and several subterminal repeats (STR), whose detailed topology is shown in the upper part of the figure. Binding sites (bs) for Abflp and Tbf1p are indicated.

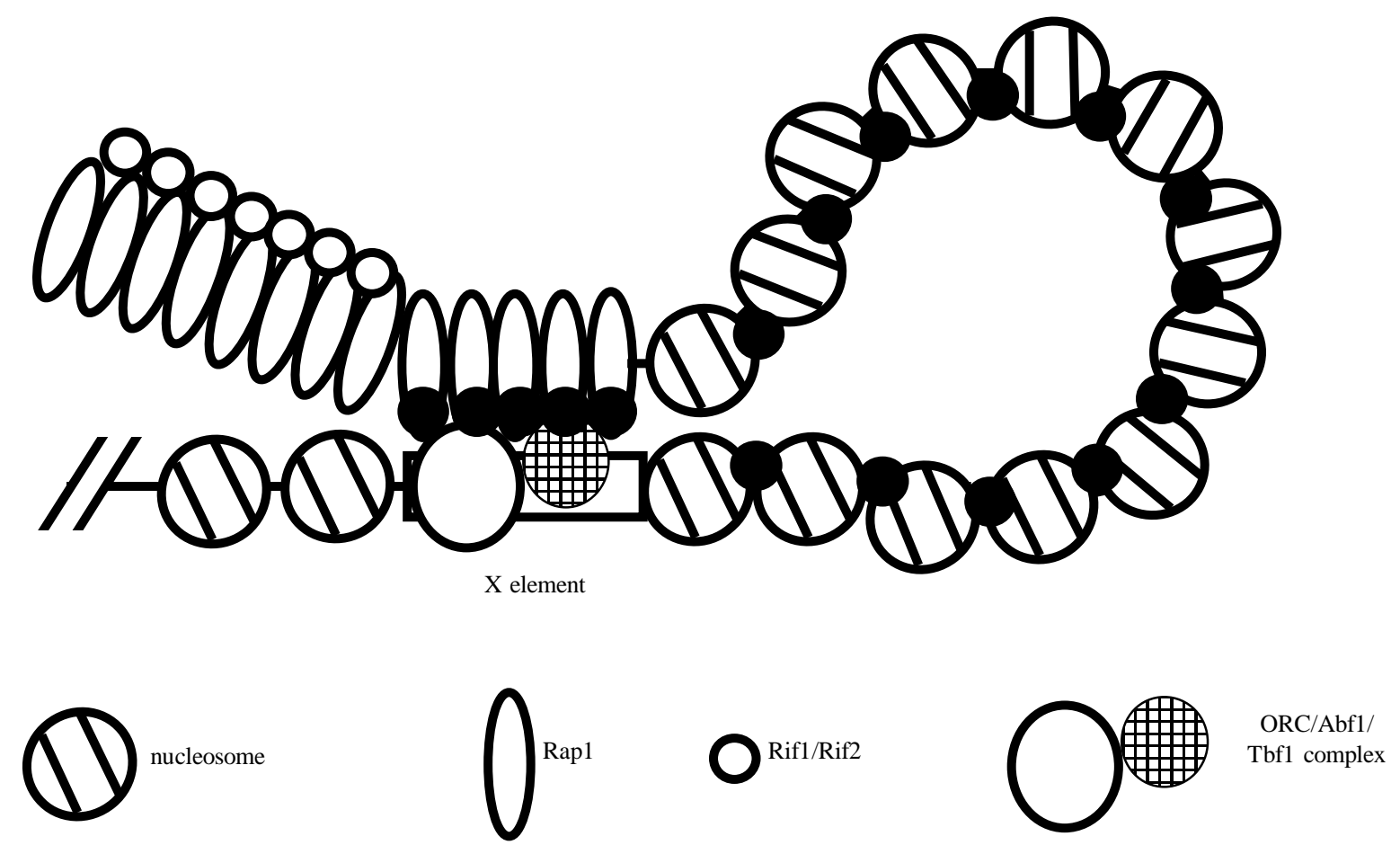

Sir complex

Fig. 4. A model of looped telomeric chromatin in Saccharomyces cerevisiae (according to Pryde and Louis 1999; see text for more details). For simplicity, only a subset of telomeric proteins is shown. 


\section{LOOPING AS A COMMON THEME IN TELOMERE ARCHITECTURE I: THE BUDDING YEAST}

S. cerevisiae with its powerful genetic and molecular biology tools seems to be a strong candidate for detailed analysis of t-loop formation. However, it seems unlikely that the budding yeast telomeres form 'true' t-loops. For example, telomeric sequences in $S$. cerevisiae are very heterogeneous $\left(\mathrm{C}_{2-3} \mathrm{ACA}_{1-6} / \mathrm{T}_{1-6} \mathrm{GTG}_{2-3}\right)$ (Shampay et al. 1984, Wang and Zakian 1990), decreasing the probability of invasion of the 3' telomeric overhang into the double-stranded telomeric region based on nucleotide complementarity. In addition, the budding yeast genome does not contain a gene encoding a TRF-like telomeric protein. The only known ortholog of a human telomeric protein scRap1p, containing its own Myb domain - seems to fulfill functions divided between TRF proteins and hRap1p in mammalian cells (Marcand et al. 1997, McEachern et al. 2000).

Based on a presence of two repetitive sequences ( $\mathrm{X}$ and $\mathrm{Y}^{\prime}$ ), the chromosome ends in S. cerevisiae are generally of one of two types (Fig. 3): those that have only $\mathrm{X}$ elements and those that have $\mathrm{X}$ and $\mathrm{Y}^{\prime}$ elements. The $\mathrm{X}$ element can now be defined as a core $\mathrm{X}$ component along with associated variable sequences. The core $\mathrm{X}$ is found in some form at all 32 ends. All ends contain at least the X-ACS region and 31 of the 32 ends contain the conserved Abf1p binding site. Most of the core X elements are full length (approximately 475 bp). Between the $X$ elements and the terminal telomere repeats (or between the $\mathrm{X}$ and $\mathrm{Y}^{\prime}$ elements) are several small subtelomeric repeat sequences (STRs) that can vary in presence/absence as well as copy number. These contain potential binding sites for Tbflp. The $\mathrm{Y}^{\prime}$ elements can vary in copy number from 1-4 tandem repeats when present at an end and usually have telomeric repetitive sequences at the centromere proximal end (see the web page of Ed Louis at http://www.le.ac.uk/ge/ej112/ for more details).

Indirect genetic evidence suggests that telomeres in $S$. cerevisiae appear to form fold-back structures thus emphasizing the role of telomere looping as a common theme in telomere architecture (de Bruin et al. 2000, de Bruin et al. 2001) (Fig. 4). These results support previous observations from Grunstein's laboratory proposing that the folding back of telomeric DNA allows interaction between Sir protein complexes bound to Rap1p, and those associated with the more internal histones (Grunstein 1997). These complexes form core heterochromatin which can spread through interactions between Sir3p and the histones. A similar looping model has been proposed to explain silencing in Drosophila, with interactions between Polycomb group proteins leading to the formation of a core silencing complex (Pirrotta 1997). In an alternative model, Pryde and Louis proposed that silencing at native ends requires the interaction of telomere-associated Rap1p/Sir2p,3p,4p complexes with proteins bound at subtelomeric, $\sim 450 \mathrm{bp}$ repetitive sequence (called $\mathrm{X}$ element), leading to the formation of a region of highly repressive chromatin (Pryde and Louis, 1999). To directly visualize the telomeric structure, we developed a system based on a $S$. cerevisiae minichromosome carrying an array of lac operator sequences. To isolate the minichromosome in a sufficient quantity and purity we took advantage of a system provided by Robert Simpson from Pennsylvania State University. The system is based on a lac repressor cloned in fusion with a chitin-binding domain separated with an intein sequence. The lac repressor-fusion protein is purified from the bacteria by chitin-sepharose thus making an affinity matrix for purification of the lac operator containing DNA. The bound DNA is eluted from the beads by DTT-induced intein splicing leading to the fractions that can be checked for purity of the preparation by PCR and inspected by EM. The system should be instrumental not only for visualization of the telomeric structure, but in the long run also for assessment of the positions of candidate telomeric proteins within the budding yeast telosome.

\section{LOOPING AS A COMMON THEME IN TELOMERE ARCHITECTURE II: THE FISSION YEAST}

In contrast to $S$. cerevisiae, fission yeast contains a TRF homologue (Taz1p) and similarly to mammalian cells, spRap1 binds to the telomeres indirectly via Taz1p. In addition, the role of spRap1p seems to be restricted to telomeres and thus is distinct from that of scRaplp, which is involved in silencing at both the telomere and mating type locus (Park et al. 2002). Therefore, fission yeast is a suitable model for the study of TRF-mediated telomere transactions and evolution of mammalian-like telosome.

Tazlp (telomere-associated in Schizosaccharomyces pombe) was originally found in a one-hybrid screen using telomeric DNA as a target (Cooper et al. 1997). It shares homology to the Myb proto-oncogene DNA-binding domain with both TRF1 and TRF2. In addition, similar to TRF1 and TRF2, it contains a centrally located sequence motif of about 200 amino acids, referred to as the TRF homology (TRFH) domain that is 
unique to this gene family and overlaps with a dimerization domain mediating strong homotypic interactions (Bianchi et al. 1997, Broccoli et al. 1997). The possibility that Taz1p functions in fission yeast may encompass those of both hTRF1 and hTRF2 is consistent with the observation that the Myb domain and TRFH domain of Taz1p are more homologous to hTRF1, whereas other regions of Taz1 show higher levels of homology with hTRF2 (Li et al. 2000).

Disruption of the $t a z 1^{+}$gene in S. pombe is not lethal and tazl haploids grow vegetatively at the same rate as the wild-type cells. Deletion of $t a z 1^{+}$ causes a dramatic (about 10-fold) increase in telomere length (Cooper et al. 1997). However, despite the changes in telomere length, tazl ${ }^{-}$cells did not exhibit a decrease in viability after extensive sub-culturing (Cooper et al., 1997). In contrast to the vegetative growth, sexual reproduction of $\operatorname{tazl}^{-}$cells is aberrant due to defective meiosis (Cooper et al. 1997, Cooper et al. 1998, Nimmo et al. 1998)). Detailed analysis of the role of Taz1p in meiotic division revealed that it is necessary for telomere aggregation adjacent to the spindle pole body (SPB) during meiotic prophase. In addition to its role in meiosis, Taz1p is required for the repression of telomere-adjacent gene expression (Cooper et al. 1997), for telomere protection under nitrogen starvation inducing an uncommitted G1 state (Ferreira and Cooper 2001), and for cell cycle progression at $20^{\circ} \mathrm{C}$, a temperature at which $\Delta$ tazl mutants exhibit a G2/M checkpoint delay, chromosome missegregation and DNA double-strand breaks (Miller and Cooper 2003). Taz1p thus represents a telomeric cap that prevents non-homologous endjoining machinery from recognizing telomeres as double-strand breaks.

Similar to the $\operatorname{tazl}^{-}$mutant, the $\mathrm{rapl}^{-}$mutant of $S$. pombe is nonessential for mitotic growth, but leads to elongation of telomeres and defective meiosis. Cells disrupted for both $\operatorname{tazl}^{+}$and $\mathrm{rapl}^{+}$ genes exhibit the same phenotype as single disruptants, suggesting that they function in the same pathway(s) (Chikashige and Hiraoka 2001). Thus Raplp of fission yeast binds to the telomere through interaction with Taz1p, as found in human cells (Li et al., 2000).

In addition to Taz1p and Rap1p, and similar to higher eukaryotes, other proteins, such Pot1p, Est1p, Ku, and Mre11/Nbs1/Rad50 complex are involved in a formation of higher-order telomere structure in the fission yeast. It is in our interest to assess the direct role of these telomeric components in the formation of fission yeast telosome. However, the size of fission yeast telomeric tracts $(<500 \mathrm{bp}$ in $S$. pombe versus $>5 \mathrm{~kb}$ in mammalian cells) and the low abundance of telomeres (6 telomeres per fission yeast cell versus 92 telomeres per human diploid cell) exclude the possibility of quantitatively isolating $S$. pombe telomeric fragments and thus assaying for the presence of their structure in vivo analogously to mammalian cells (Griffith et al. 1999). Therefore we are trying to reconstitute the fission yeast telosome using a model telomere DNA containing a double-stranded array of fission yeast telomeric repeats in which the single-stranded overhang can be altered in sequence and length. Our ultimate goal is to reconstitute the fission yeast telosome in vitro from purified components. We have initiated a study of the ability of recombinant Taz1p to bind and to remodel the fission yeast telomere in vitro (Fig. 2C). We believe that the results will provide the first step towards understanding telomere remodeling in fission yeast that, in addition to its evolutionary implications, might be relevant for the understanding of the molecular principles of telomere dynamics in higher eukaryotes.

\section{FINAL REMARKS}

In the current textbooks of molecular biology, telomeres are described as structures at the ends of linear DNA molecules required for their complete replication. In addition to their role in DNA synthesis, telomeres must protect the chromosome ends from DNA damage responses such as cell cycle arrest and apoptosis. This feature of telomeres is especially important for cancer cells, which continue proliferating in spite of chromosome aberrations. The telomere capping is probably mediated by several factors acting in parallel, including an active (and novel) role for (i) telomerase, (ii) DNA damage proteins involved in non-homologous end-joining pathway and (iii) overall telomere architecture. The latter mechanism of telomere protection is exemplified by telomere looping represented either by invasion of a 3 ' telomeric overhang into the duplex region of the telomere (t-loops), or by fold-back structures induced by protein-protein interactions at the telomere. We have developed experimental systems to study both mechanisms in fission and budding yeasts, respectively, with the ultimate goal of answering the question in the title of our presentation.

\section{ACKNOWLEDGEMENTS}

We wish to thank the members of our laboratories for discussions and L. Kovac (Department of 
Biochemistry, Comenius University, Bratislava) for helpful comments and continuous support.

This work was supported by Fogarty International Research Collaboration Award (1-R03-TW0565401, CFDA No. 93.934), Howard Hughes Medical Institute Grant (55000327), and grants from the Slovak grant agency VEGA (1/9153/02, 1/0006/03) and APVT (20-003902) and grant from the Comenius University (UK/131/2002).

Received 30 $0^{\text {th }}$ May 2003.

Published online $30^{\text {th }}$ June 2003.

Simultaneously published in J. Berger (ed.): Advances in Cell Biology. Kopp Publ., České Budějovice, pp. 3959, 2003.

\section{REFERENCES}

Autexier C. and C.W. Greider: Telomerase and cancer: revisiting the telomere hypothesis. Trends Biochem. Sci. 21: 387-391, 1996.

Baumann P. and T.R. Cech: Pot1, the putative telomere end-binding protein in fission yeast and humans. Science 292: 1171-1175, 2001.

Bianchi A., S. Smith, L. Chong, P. Elias, T. de Lange: TRF1 is a dimer and bends telomeric DNA. EMBO J. 16: 1785-1794, 1997.

Biessmann H. and J.M. Mason: Telomere maintenance without telomerase. Chromosoma 106: 63-69, 1997.

Bilaud T., C. Brun, K. Ancelin, C.E. Koering, T. Laroche, E. Gilson: Telomeric localization of TRF2, a novel human telobox protein. Nat. Genet. 17: 236-239, 1997.

Blackburn E.H.: Telomere states and cell fates. Nature 408: 53-56, 2000.

Blasco M.A., H.W. Lee, M.P. Hande, E. Samper, P.M. Lansdorp, R.A. DePinho, C.W. Greider: Telomere shortening and tumor formation by mouse cells lacking telomerase RNA. Cell 91: 25-34, 1997.

Bodnar A.G., M. Ouellette, M. Frolkis, S.E. Holt, C.P. Chiu, G.B. Morin, C.B. Harley, J.W. Shay, S. Lichtsteiner, W.E. Wright: Extension of lifespan by introduction of telomerase into normal human cells. Science 279: 349-352, 1998.

Broccoli D., A. Smogorzewska, L. Chong, T. de Lange: Human telomeres contain two distinct Myb-related proteins, TRF1 and TRF2. Nat. Genet. 17: 231-235, 1997.

Bryan T.M., A. Englezou, L. Dalla-Pozza, M.A. Dunham, R.R. Reddel: Evidence for an alternative mechanism for maintaining telomere length in human tumors and tumor-derived cell lines. Nat. Med. 3: 1271-1274, 1997.

Bryan T.M., A. Englezou, J. Gupta, S. Bacchetti, R.R. Reddel: Telomere elongation in immortal human cells without detectable telomerase activity. EMBO J. 14: 4240-4248, 1995.

Bryan T.M., L. Marusic, S. Bacchetti, M. Namba, R.R. Reddel: The telomere lengthening mechanism in telomerase-negative immortal human cells does not involve the telomerase RNA subunit. Hum. Mol. Genet. 6: 921-926, 1997.

Bryan T.M. and R.R. Reddel: Telomere dynamics and telomerase activity in in vitro immortalised human cells. Eur. J. Cancer 33: 767-773, 1997.

Chan S.W.-L. and E.H. Blackburn: New ways not to make ends meet: telomerase, DNA damage proteins and heterochromatin. Oncogene 21: 553563, 2002.

Chikashige Y. and Y. Hiraoka: Telomere binding of the Rap1 protein is required for meiosis in fission yeast. Curr. Biol. 11: 1618-1623, 2001.

Chong L., B. van Steensel, D. Broccoli, H. Erdjument-Bromage, J. Hanish, P. Tempst, T. de Lange: A human telomeric protein. Science 270: 1663-1667, 1995.

Colgin L.M. and R.R. Reddel: Telomere maintenance mechanisms and cellular immortalization. Curr. Opin. Genet. Dev. 9: 97103, 1999.

Cooper J.P., E.R. Nimmo, R.C. Allshire, T.R. Cech: Regulation of telomere length and function by a Myb-domain protein in fission yeast. Nature 385: 744-747, 1997.

Cooper J.P., Y. Watanabe, P. Nurse: Fission yeast Taz1 protein is required for meiotic telomere clustering and recombination. Nature 392: 828831, 1998.

de Bruin D., S.M. Kantrow, R.A. Liberatore, V.A. Zakian: Telomere folding is required for the stable maintenance of telomere position effects in yeast. Mol. Cell. Biol. 20: 7991-8000, 2000.

de Bruin D., Z. Zaman, R.A. Liberatore, M. Pashne: Telomere looping permits gene activation by a downstream UAS in yeast. Nature 409: 109-113, 2001.

Desmaze C., J.C. Soria, M.A. Freulet-Marriere, N. Mathieu, L. Sabatier: Telomere-driven genomic instability in cancer cells. Cancer Lett. 194: 173-182, 2003.

Dunham M.A., A.A. Neumann, C.L. Fasching, R.R. Reddel: Telomere maintenance by recombination in human cells. Nat. Genet. 26: 447-450, 2000.

Evans S.K. and V. Lundblad: Est1 and Cdc13 as comediators of telomerase access. Science 286 : 117-120, 1999. 
Ferreira M.G. and J.P. Cooper: The fission yeast Taz1 protein protects chromosomes from $\mathrm{Ku}-$ dependent end-to-end fusions. Mol. Cell. 7: 5563, 2001.

Greider C.W.: Telomerase activation: One step on the road to cancer? Trends Cell. Biol. 15: 109112, 1999.

Griffith J.D., A. Bianchi, T. de Lange: TRF1 promotes parallel pairing of telomeric tracts in vitro. J. Mol. Biol. 278: 79-88, 1998.

Griffith J.D., L. Comeau, S. Rosenfield, R.M. Stansel, A. Bianchi, H. Moss, T. de Lange: Mammalian telomeres end in a large duplex loop. Cell 97: 503-514, 1999.

Grunstein M.: Molecular model for telomeric heterochromatin in yeast. Curr. Opinion Cell Biol. 9: 383-387, 1997.

Hahn W.C., S.A. Stewart, M.W. Brooks, S.G. York, E. Eaton, A. Kurachi, R.L. Beijersbergen, J.H. Knoll, M. Meyerson, R.A. Weinberg: Inhibition of telomerase limits the growth of human cancer cells. Nat. Med. 5: 1164-1170, 1999.

Holt S.E., J.W. Shay, W.E. Wright: Refining the telomere-telomerase hypothesis of aging and cancer. Nat. Biotechnol. 14: 836-839, 1996.

Karlseder J.: Telomere repeat binding factors: keeping the ends in check. Cancer Lett. 194: 189-197, 2003.

Karlseder J., A. Smogorzewska, T. de Lange: Senescence induced by altered telomere state, not telomere loss. Science 295: 2446-2449, 2002.

Kim S.H., P. Kaminker, J. Campisi: TIN2, a new regulator of telomere length in human cells. Nat. Genet. 23: 405-412, 1999.

Lansdorp P.M.: Lessons from mice without telomerase. J. Cell. Biol. 139: 309-312, 1997.

Levis R.W., R. Ganesan, K. Houtchens, L.A. Tolar, F.M. Sheen: Transposons in place of telomeric repeats at a Drosophila telomere. Cell 75: 10831093, 1993.

Li B., S. Oestreich, T. de Lange: Identification of human Rap1: implications for telomere evolution. Cell 101: 471-483, 2000.

Marcand S., E. Gilson, D. Shore: A proteincounting mechanism for telomere length regulation in yeast. Science 275: 986-990, 1997.

McEachern M.J. and E.H. Blackburn: Capprevented recombination between terminal telomeric repeat arrays (telomere CPR) maintains telomeres in Kluyveromyces lactis lacking telomerase. Genes Dev. 10: 1822-1834, 1996.

McEachern M.J., S. Iyer, T.B. Fulton, E.H. Blackburn: Telomere fusions caused by mutating the terminal region of telomeric DNA. Proc. Natl. Acad. Sci. USA 97: 11409-11414, 2000.
McEachern M.J., A. Krauskopf, E.H. Blackburn: Telomeres and their control. Ann. Rev. Genet. 255: 331-358, 2000.

Miller K.M. and J.P. Cooper: The telomere protein Taz1 is required to prevent and repair genomic DNA breaks. Mol. Cell 11: 303-313, 2003.

Munoz-Jordan J.L., G.A. Cross, T. de Lange, J.D. Griffith: t-loops at trypanosome telomeres. EMBO J. 20: 579-588, 2001.

Murti K.G. and D.M. Prescott: Telomeres of polytene chromosomes in a ciliated protozoan terminate in duplex DNA loops. Proc. Natl. Acad. Sci. USA 96: 14436-14439, 1999.

Naito T., A. Matsuura, F. Ishikawa: Circular chromosome formation in a fission yeast mutant defective in two ATM homologues. Nat. Genet. 20: 203-206, 1998.

Nakamura T.M., J.P. Cooper, T.R. Cech: Two modes of survival of fission yeast without telomerase. Science 282: 493-496, 1998.

Nimmo E.R., A.L. Pidoux, P.E. Perry, R.C. Allshire: Defective meiosis in telomere-silencing mutants of Schizosaccharomyces pombe. Nature 392: 825-828, 1998.

Pardue M.L., O.N. Danilevskaya, K. Lowenhaupt, F. Slot, K.L. Traverse: Drosophila telomeres: new views on chromosome evolution. Trends Genet. 12: 48-52, 1996.

Pardue M.L., O.N. Danilevskaya, K.L. Traverse, K. Lowenhaupt: Evolutionary links between telomeres and transposable elements. Genetica 100: 73-84, 1997.

Park M.J., Y.K. Jang, E.S. Choi, H.S. Kim, S.D. Park: Fission yeast Rap1 homolog is a telomere-specific silencing factor and interacts with Taz1p. Mol. Cells 13: 327-333, 2002.

Pirrotta V.: Chromatin-silencing mechanisms in Drosophila maintain patterns of gene expression. Trends Genet. 13: 314-318, 1997.

Pryde F.E. and E.J. Louis: Limitations of silencing at native yeast telomeres. EMBO J. 18: 25382550, 1999.

Raymond E., D. Sun, S.F. Chen, B. Windle, D.D. Von Hoff: Agents that target telomerase and telomeres. Curr. Opin. Biotechnol. 7: 583-591, 1996.

Reddel R.R., T.M. Bryan, L.M. Colgin, K.T. Perrem, T.R. Yeager: Alternative lengthening of telomeres in human cells. Radiat. Res. 155: 194-200, 2001.

Reddel R.R., T.M. Bryan, J.P. Murnane: Immortalized cells with no detectable telomerase activity. Biochemistry (Moscow) 62: 1254-1262, 1997.

Rhodes D., L. Fairall, T. Simonsson, R. Court, L. Chapman: Telomere architecture. EMBO Rep. 3: 1139-1145, 2002. 
Shampay J., J.W. Szostak, E.H. Blackburn: DNA sequences of telomeres maintained in yeast. Nature 310: 154-157, 1984.

Smith S., I. Giriat, A. Schmitt, T. de Lange: Tankyrase, a poly(ADP-ribose) polymerase at human telomeres. Science 282: 1484-1487, 1998.

Smogorzewska A., B. van Steensel, A. Bianchi, S. Oelmann, M.R. Schaefer, G. Schnapp, T. de Lange: Control of human telomere length by TRF1 and TRF2. Mol. Cell. Biol. 20: 16591668, 2000.

Stansel R.M., T. de Lange, J.D. Griffith: T-loop assembly in vitro involves binding of TRF2 near the 3' telomeric overhang. EMBO J. 20: 55325540, 2001.

Tomaska L., A.M. Makhov, J.D. Griffith, J. Nosek: t-loops in yeast mitochondria. Mitochondrion 1 : 455-459, 2002.

van Steensel B. and T. de Lange: Control of telomere length by the human telomeric protein TRF1. Nature 385: 740-743, 1997.

Wang S.S. and V.A. Zakian: Sequencing of Saccharomyces telomeres cloned using T4 DNA polymerase reveals two domains. Mol. Cell. Biol. 10: 4415-4419, 1990.

Wang S.S. and V.A. Zakian: Telomere-telomere recombination provides an express pathway for telomere acquisition. Nature 345: 456-458, 1990.

\section{* Address:}

L’ubomír Tomáška, Department of Genetics, Comenius University, Faculty of Natural Sciences, Mlynská dolina B-1, 84215 Bratislava, Slovakia; tomaska@fns.uniba.sk 